\title{
Indústria do Calçado: Principais Fatores de Risco e Riscos Laborais, Doenças Profissionais associadas e Medidas de Proteção recomendadas
}

rpso.pt/industria-do-calcado-principais-fatores-risco-riscos-laborais-doencas-profissionais-associadas-medidasprotecao-recomendadas/

SHOE INDUSTRY: MAIN LABOR FACTOR RISKS AND RISKS,

ASSOCIATED PROFESSIONAL DISEASES AND

RECOMMENDED PROTECTIVE MEASURES

TIPO DE ARTIGO: Revisão Bibliográfica Integrativa

AUTORES: Santos M(1), Almeida A(2).

\section{RESUMO}

\section{Introdução/ enquadramento/ objetivos}

A indústria do calçado está bastante desenvolvida em algumas zonas do nosso país, pelo que existe um número razoável de trabalhadores envolvidos neste setor. Os principais fatores de risco/ riscos associados são o contato com agentes químicos (e eventuais patologia oncológica, respiratória, dermatológica, oftalmológica e otorrinolaringológica); as posturas mantidas/ forçadas e movimentos repetitivos (e consequentes lesões músculo-esqueléticas); esforço visual (e eventual diminuição da acuidade); ruído (realçando-se aqui a hipoacusia); utilização de máquinas capazes de proporcionar acidentes (como corte, contusão, atracamento, amputação) e vibrações (com possíveis alterações vasculares e neurológicas).

Pretende-se com esta revisão conhecer melhor os fatores de risco/ riscos existentes neste setor profissional.

\section{Metodologia}

Trata-se de uma Revisão Bibliográfica Integrativa, iniciada através de uma pesquisa realizada em novembro de 2017 nas bases de dados "CINALH plus with full text, Medline with full text, Database of Abstracts of Reviews of Effects, Cochrane Central Register of 
Controlled Trials, Cochrane Database of Systematic Reviews, Cochrane Methodology Register, Nursing and Allied Health Collection: comprehensive, MedicLatina, Academic Search Complete e RCAAP".

\section{Conteúdo}

A bibliografia sobre o setor é escassa e destaca sobretudo os agentes químicos e, secundariamente, as questões músculo-esqueléticas.

$\mathrm{Na}$ indústria do calçado as tarefas com maior contato com os solventes são a colagem e a preparação das solas, bem como a montagem e acabamento. Num estudo onde se avaliaram cerca de 100 empresas do norte do país, verificou-se que os solventes mais frequentes foram o tolueno e o n-hexano; em $58 \%$ dos casos foram encontrados valores acima dos níveis máximos recomendados pela NP 1796, pela EN 689 o pelos TLVs (threstholds limit values) da ACGIH.

\section{Conclusões}

A bibliografia consultada deu algum destaque para as alterações hematológicas (como linfomas e leucemias); neuropatias e parésias; hepatoxicidade; dermatites; insuficiência renal; asma e apneia do sono; hipoacusia; discromotopsia/ maculopatia; bem como Síndroma do Túnel Cárpico ou do Canal de Guyon, tendinites e tenossinovites, síndroma de Raynaud e doenças de Kienbock, de Quervain e Dupuytren.

Seria pertinente desenvolver investigações que avaliassem a realidade nacional.

PALAVRAS/ EXPRESSÕES- CHAVE: saúde ocupacional, saúde do trabalhador e medicina do trabalho; indústria do calçado.

\section{ABSTRACT}

\section{Introduction / framework / objectives}

The footwear industry is well developed in some areas of our country, so there is a reasonable number of workers involved in this sector. The main risk/ risk factors are the contact with chemical agents (and eventual oncological, respiratory, dermatological, ophthalmological and otorhinolaryngological pathology); as maintained/ forced postures (standing or sitting) and repetitive movements (and consequent musculoskeletal injuries); visual acuity (and eventual diminution of acuity, eye fatigue); noise (emphasizing in hypoacusis); use of machines capable of cause accidents (such as cutting, bruising, docking, amputation) and vibrations (with possible vascular and neurological changes).

It is intended with this review better understand the existing risks/ risk factors in the professional sector.

\section{Methodology}


This is an Integrative Bibliographic Review, initiated through a survey conducted in November 2017 in the databases "CINALH plus with full text, Medline with full text, Database of summaries of effects reviews, Cochrane Central Register of Controlled Trials, Cochrane Database of Systematic Reviews, Cochrane Methodology Register, Nursing and Allied Health Collection: Comprehensive, MedicLatina, Academic Search Complete and RCAAP.

\section{Content}

The bibliography on this sector is scarce and emphasizes mainly the chemical agents and, secondarily, the musculoskeletal issues.

In the footwear industry the tasks with the greatest contact with the solvents are the gluing and preparation of the soles, as well as assembly and finishing. In a study where about 100 companies from the north of the country were evaluated, the most frequent solvents were toluene and $n$-hexane; in $58 \%$ of the cases, values above the maximum levels recommended by NP 1796, EN 689 and ACGIH threshold limit values were found.

\section{Conclusions}

The literature consulted highlighted hematological alterations (as lymphomas and leukemias); neuropathies and paresis; hepatotoxicity; dermatitis; renal insufficiency; asthma and sleep apnea; hearing loss; discromotopsy / maculopathy; as well as Carpal Tunnel Syndrome or Guyon's Channel, tendinitis and tenosynovitis, Raynaud's syndrome, and Kienbock, Quervain and Dupuytren diseases.

It would be pertinent to develop investigations that evaluate the national reality.

WORDS / KEY EXPRESSIONS: occupational health, worker health and occupational medicine; footwear industry.

\section{INTRODUÇÃO}

A indústria do calçado está bastante desenvolvida em algumas zonas do nosso país, pelo que existe um número razoável de trabalhadores envolvidos neste setor. Os principais fatores de risco/ riscos associados são o contato com agentes químicos (e eventuais patologia oncológica, respiratória, dermatológica, oftalmológica e otorrinolaringológica); as posturas mantidas/ forçadas e movimentos repetitivos (e consequentes lesões músculo-esqueléticas); esforço visual (e eventual diminuição da acuidade, cansaço ocular); ruído (realçando-se aqui a hipoacusia); utilização de máquinas capazes de proporcionar acidentes (como corte, contusão, atracamento, amputação) e vibrações (com possíveis alterações vasculares e neurológicas).

\section{METODOLOGIA}


Pergunta protocolar: quais os principais riscos e fatores de risco existentes para os profissionais que trabalham na indústria do calçado, eventuais doenças profissionais associadas e medidas de proteção recomendadas?

Em função da metodologia PICo, foram considerados:

-P (population): funcionários a exercer em empresas produtoras de calçado.

-I (interest): reunir conhecimentos relevantes sobre os principais riscos e fatores de risco existentes para os profissionais que exercem na indústria do calçado, eventuais doenças profissionais associadas e medidas de proteção recomendadas, segundo os dados mais recentemente publicados

-C (context): saúde ocupacional nas empresas deste setor

Foi realizada uma pesquisa em novembro de 2017 nas bases de dados "CINALH plus with full text, Medline with full text, Database of Abstracts of Reviews of Effects, Cochrane Central Register of Controlled Trials, Cochrane Database of Systematic Reviews, Cochrane Methodology Register, Nursing and Allied Health Collection: comprehensive, MedicLatina e Academic Search Complete". Utilizando as palavras-chave "shoe e footwear" foram obtidos 773 e 519 artigos; conjugando a primeiras destas com "occupational" e a segunda com "industry" foram obtidos 65 e 9 trabalhos, respetivamente, com os critérios publicação igual ou superior a 2007 e acesso a texto completo; foram selecionados após a leitura do resumo 9 e 2 artigos e, após a consulta do trabalho na íntegra, 5 e 2 artigos.

Contudo, como não se encontraram estudos relativos à realidade portuguesa nestas bases de dados indexadas, os autores procuraram trabalhos inseridos no RCAAP (Repositório Científico de Acesso Aberto em Portugal). Aqui, utilizando as palavras/ expressões-chave "indústria do calçado e calçado+ saúde ocupacional", foram obtidos 240 e 5 documentos; após a leitura do resumo dos mesmos foram selecionadas 5 e 2 investigações; após a consulta na íntegra selecionou-se um artigo em cada, que correspondia ao mesmo trabalho (repetição).

O resumo da metodologia aplicada nesta revisão pode ser consultado nos fluxogramas de $1^{\mathrm{a}}$ e $2^{\mathrm{a}}$ fases. A caraterização metodológica de cada trabalho pode ser consultada na Tabela 1.

\section{CONTEÚDO}

A nível internacional tem-se verificado um deslocamento investimento de algumas empresas deste setor para países não desenvolvidos ou em vias de desenvolvimento, onde o cumprimento/ existência de normas de saúde ocupacional poderá ser mais rudimentar que nos países mais desenvolvidos ${ }^{1}$. 
No Brasil, por exemplo, em 2006, estimava-se a existência de cerca de 9500 empresas produtoras de calçado, que empregavam cerca de 212 mil indivíduos. Na generalidade dos casos o processo produtivo estava organizado em função dos princípios de Taylor e Ford ${ }^{2,3}$ onde, através da fragmentação da tarefa e especialização do funcionário, se obteria maior produtividade; contudo, presentemente sabe-se que tal simultaneamente implica mais danos médicos e pior desempenho laboral ${ }^{2}$. Logo, a taxa de acidentes é elevada, tal como o absentismo e o turnover entre operários. Outros autores destacam que em 2007 este país era responsável por cerca de 5\% da produção mundial de calçado. Em 2010 estes estimaram que existiram cerca de 8200 empresas deste setor, empregando cerca de 349.000 trabalhadores $^{3}$.

Considera-se que a Indonésia é o terceiro maior exportador de calçado ${ }^{1}$, antecedido pela China e pelo Vietname ${ }^{1,4}$. Aliás, em 2009, estimou-se que cerca de 115.000 de indivíduos estavam empregues nesta área de trabalho, por vezes subcontratados por grandes empresas multinacionais europeias, por exemplo ${ }^{1}$. No Vietname são empregues anualmente cerca de 2,5 milhões de trabalhadores, $85 \%$ dos quais pertencentes ao sexo feminino ${ }^{4}$.

Contudo, a bibliografia sobre o setor é escassa e destaca sobretudo os agentes químicos e, secundariamente, as questões músculo-esqueléticas.

\section{Agentes químicos}

O baixo peso molecular e a lipofilicidade promovem uma rápida absorção cutânea dos solventes; estes são por isso facilmente armazenados no tecido adiposo ${ }^{5}$.

A principal via de eliminação é a hepática, através do citocromo P450 (no caso específico do benzeno geram-se aqui metabolitos muito tóxicos) ${ }^{5}$.

Muitos solventes são COVs (compostos orgânicos voláteis) que se definem como sendo qualquer substância com carbono que participe em reações atmosféricas fotoquímicas (à exceção do monóxido e dióxido de carbono, ácido carbónico, carbonetos, carbonatos metálicos e carbonato de amónia); ainda que as definições variem muito entre países e instituições. A nível ocupacional a principal via de entrada dos COVs é a respiratória ${ }^{5}$.

Um solvente orgânico será um agente líquido entre os zero e os $250^{\circ}$, volátil e relativamente inerte quimicamente, de forma a conseguir extrair, dissolver ou suspender substâncias não solúveis na água (como gorduras, resinas e polímeros) ${ }^{5}$.

Entre os solventes orgânicos mais usados a nível laboral global destacam-se os hidrocarbonetos aromáticos (benzeno, tolueno, xileno), hidrocarbonetos alifáticos (nhexano), hidrocarbonetos clorados (diclorometano, clorobenzeno, tetracloroetileno, álcoois, ésteres de ácidos carboxílicos, cetonas, éteres de glicol e amidas). Na indústria do calçado as tarefas com maior contato com os solventes são a colagem e a preparação das solas, bem como montagem e acabamento. Num estudo onde se avaliaram cerca de 100 empresas do norte do país, verificou-se que os solventes mais 
frequentes foram o tolueno e o n-hexano; em $58 \%$ dos casos foram encontrados valores acima dos níveis máximos recomendados pela NP 1796, pela EN 689 o pelos TLVs (threstholds limit values) da $\mathrm{ACGIH}^{5}$.

No caso do n-hexano a biomonitorização poderá ser realizada através do doseamento da 2,5 hexadiona, uma vez que se trata do metabolito deste agente mais tóxico no contexto neurológico 5 .

Os efeitos agudos globais incidem nas alterações do SNC (Sistema Nervoso Central), como tonturas, sonolência e apatia, ainda que possa ocorrer a morte. O n-hexano e o metil-n-butilcetona podem originar uma neuropatia periférica progressiva com diminuição da sensibilidade distal e eventual parésia. O benzeno pode levar a aplasia medular. Já o tetracloreto de carbono, clorofórmio e dimetilformamida podem justificar hepatotoxicidade. Por sua vez, no sistema reprodutor, são salientados o óxido de etileno e os éteres de glicol. Vários destes agentes também causam dermatites e até patologia oncológica (leucemias, linfomas, mieloma múltiplo). O tolueno poderá causar alterações nos túbulos renais. A principal semiologia respiratória mencionada na bibliografia é a tosse, dispneia, pieira, expetoração; são também mais prevalentes a asma e a apneia o sono, segundo alguns autores ${ }^{5}$.

\section{-Destaque ao Benzeno}

Apesar de entre os artigos selecionados para esta revisão não serem mencionados mais dados relevantes sobre o benzeno, os autores gostariam de realçar alguns aspetos, provenientes de uma outra revisão realizada, relativa aos postos de abastecimento de combustíveis, também publicada nesta revista, no final de novembro de 2017 (volume 4).

Aí mencionou-se que este é uma substância classificada como carcinogénica para humanos (grupo I), pela IARC (International Agency for Research on Cancer), desde 1982 e pela United States Environmental Protection Agency (USEPA). Os limites de exposição podem variar entre países e agências, ainda que, simultaneamente, não exista limite abaixo do qual se considera que um agente cancerígeno é seguro. $\mathrm{O}$ diagnóstico da intoxicação é basicamente clínico, associando a exposição à semiologia (sinais e sintomas) e, posteriormente, aos exames laboratoriais.

Os seus metabolitos podem interferir com o ciclo celular, induzindo apoptose das células percursoras hematopoiéticas e alterações nas vias de sinalização celular.

A quantificação do benzeno pode ser realizada através da monitorização ambiental e por biomonitorização (do benzeno em si e dos seus metabólitos). Pode ser medido no ar expirado, sangue ou urina; no caso dos metabólitos só a nível urinário e após 24 horas de exposição. A principal via de entrada no organismo para o benzeno é a inalatória, ainda que tal possa ocorrer via oral e cutânea. É primariamente metabolizado no fígado e posteriormente excretado na urina; ainda que a maioria do benzeno não metabolizado seja eliminado via pulmonar. O processo de biotransformação é complexo e decorre sobretudo a nível hepático; de forma mais discreta também a nível medular. 
90\% do benzeno absorvido é excretado via urinária através de metabólitos: ácido sfenilmercaptúrico (AFM), ácido trans-trans-mucónico (AttM), fenol (PH), catecol (CA) e a hidroquinona (HG). Entre os diversos biomarcadores de exposição ao benzeno, o AFM é considerado o mais específico; contudo, devido à sua baixa concentração urinária, são necessárias técnicas mais sensíveis para a sua correta quantificação, nomeadamente a cromatografia e a espectrometria de massas, ambas complexas e dispendiosas.

Contudo, o AttM é o metabolito mais usado devido à maior facilidade técnica, ainda que a sua concentração urinária possa ser influenciada pela dieta (nomeadamente pelo sorbitol ou ácido sórbico, utilizados como conservantes alimentares). Na formação do AFM interagem enzimas como a GST (glutationa S-transferase). Este biomarcador deteta níveis urinários com exposição ao benzeno no ar inferiores a 1 ppm.

Nos EUA o valor-limite recomendado era de 100 ppm em 1946 pela ACGIH; em 1948 passou para 50 ppm e em 1976 voltou a reduzir para metade; sendo que em 1987 se adotou 1 ppm e, dez anos depois, 0,5 ppm. Já a OSHA definiu 10 ppm em 1974 e 1 ppm em 1987 ou, quando muito, 5 ppm (para exposições breves), por exemplo. A US Occupational Safety and Health Administration defende que um nível máximo de exposição de 1 ppm para turnos de oito horas e 5 ppm para períodos de quinze minutos. Contudo, estima-se que níveis na ordem das 4 ppm possam originar um caso de patologia oncológica em 10.000 expostos.

Quando a concentração de exposição é baixa ele pode ser metabolizado por duas vias distintas, o que, paradoxalmente, aumenta a toxicidade; ou seja, ele é metabolizado de forma mais eficiente em baixas concentrações, devido à diferente saturação das enzimas envolvidas; logo, haverá maior risco hematológico com níveis baixos.

A semiologia aguda é sobretudo neurológica, salientando-se as alterações da atenção e memória, cefaleia, depressão, insónia, agitação, polineuropatias, hipoacusia, acufeno e vertigem. Podem também verificar-se dermatite irritativa e mialgias. É também possível a ocorrência de hepatotoxicidade.

A semiologia associada a exposição crónica carateriza-se por mielotoxicidade (alterações quantitativas e/ou da qualidade das células sanguíneas), como já se mencionou. Aliás, com exposição prolongada pode ocorrer hipoplasia medular e pancitopenia (diminuição de todas as linhagens) - logo, o hemograma é fundamental, de fácil acesso e execução. Contudo, acredita-se que, em função da extensão da lesão, os danos poderão ter alguma reversibilidade. O benzeno também consegue alterar a robustez dos eritrócitos (colocando-os mais suscetíveis à hemólise) e alterar o seu tamanho e forma.

Existem estudos que alegam ter encontrado alterações nos espermatozoides associadas a exposições inferiores a 1 ppm, bem como aneuploidias nos cromossomas x, y e 21 .

Alguns investigadores defendem que a exposição a solventes orgânicos em geral (mesmo que em baixas concentrações) aumenta o risco de hipoacusia; tendo o ruído em simultâneo um efeito sinérgico: nomeadamente um risco cinco vezes superiores de desenvolver hipoacusia com exposição dupla versus três vezes superior quando a 
exposição é apenas ao ruído. Por vezes a acuidade parece normal se testada em silêncio, mas em ambientes ruidosos pode existir limitação de perceção da fala e deteção da fonte do som. Considera-se que o primeiro alvo dos solventes são as células ciliadas do órgão de corti; posteriormente as modificações processadas podem permitir a entrada de energia acústica mais elevada para a cóclea. Daí que alguns defendam que o limite para usar proteção auricular nos trabalhadores expostos ao ruído e a solventes devesse baixar para $80 \mathrm{~dB}(\mathrm{~A}) \mathrm{s}$.

Os trabalhadores expostos ao benzeno apresentam algumas anormalidades visuais, com realce para a descriminação das cores e alterações no campo de visão. Uns destacam que é mais frequente a perda discriminativa do azul-amarelo, ainda que outros também mencionem o vermelho-verde. A discromatopsia é atribuída à maculopatia secundária aos danos nos fotorrecetores em cone, bem como nas células ganglionares e desmielinização do nervo ótico.

\section{Contexto dermatológico}

No contexto dermatológico, este setor apresenta várias substancias com capacidade para provocarem alergias e/ou irritações cutâneas, com particular destaque para as colas, conservantes, resinas acrílicas, couro, borracha e corantes. A exposição a estes agentes químicos é particularmente mais relevante nas tarefas de colagem e limpeza; contudo, em muitas empresas, não há uma separação eficaz entre os diversos postos de trabalho, pelo que a generalidade dos funcionários contata com estes produtos, ainda que em intensidades diferentes e pelo menos por via inalatória (para os que não tocam no produto). Para além disso, a reatividade dermatológica poderá ficar potenciada pelas temperaturas elevadas e/ou humidade ${ }^{1}$.

Um estudo italiano, por exemplo, quantificou uma prevalência de dermatite de contato na ordem dos $15 \%$, hiperqueratose digital em $6 \%$ e prurido em $3 \%$; no global, cerca de um quarto dos trabalhadores apresentava alguma alteração dermatológica associada ao trabalho, situação essa parcialmente justificada pelos investigadores como estando associada ao uso escasso ou inadequado dos equipamentos de proteção individual (EPIs) ${ }^{1}$.

\section{Contexto músculo-esquelético}

Os trabalhadores a exercer neste setor apresentam risco considerável de desenvolver patologia músculo-esquelética, sobretudo em empresas menos mecanizadas e/ou mais pequenas, com destaque para as posturas mantidas/ forçadas, movimentos repetitivos e pouca valorização da ergonomia ${ }^{6,7}$, onde mais precários ou até inexistentes se tornam os serviços de saúde ocupacional ${ }^{6}$. 0 aumento da tecnologia e da produtividade poderá contribuir para uma maior especialização do trabalhador e repetição de tarefas, que poderá justificar uma diminuição do desempenho e potenciar os custos médicos e sociais (associados à dor, astenia e edema) ${ }^{7}$. 
De forma muito genérica, alguns autores recomendam a existência de assentos ajustáveis em altura, de forma a proporcionar uma postura natural, forrados de material adequado e giratórios (para atenuar os movimentos lesivos na coluna), bem como um apoio de pés ${ }^{6}$.

Alguns autores consideram que a existência de quistos na mão poderá estar associada a movimentos repetitivos e/ou vibrações. Existem duas teorias explicativas: a sobrecarga na cartilagem e consequente degeneração, combinada com o aumento da pressão intraarticular, o que faz com que o líquido sinovial extravase e seja reabsorvido a nível trabecular; outros também defendem a possibilidade de que o atrito entre superfícies ósseas não revestidas por cartilagem, associado a diminuição da vascularização, possa contribuir para o aumento da necrose óssea.

De forma mais direta estes fatores de risco podem contribuir para a síndroma de vibração mão-braço, caraterizado pelo comprometimento vascular (dedo branco induzido pela vibração, síndroma de Raynaud) e neuropatia periférica sensorial (com alteração das sensibilidade térmica e tátil) ou até mesmo síndroma do túnel cárpico, síndroma de canal de Guyon, tendinite, tenossinovite, doença de Quervain, de Dupuytren e de Kienbock ${ }^{8}$.

\section{Questões psicossociais}

Um bom ambiente de trabalho proporcionado pelas chefias e colegas de trabalho permite a criação de uma rede mais sólida de apoio, de forma a que o trabalhador consiga lidar melhor com as questões mais stressantes das suas tarefas laborais (como carga de trabalho, falta de controlo na gestão/ elaboração das tarefas, necessidade de cumprir prazos/ produtividades agressivos e/ou turnos prolongados), diminuindo o risco de alterações emocionais (como a depressão) e físicas (como a síndroma metabólica, com destaque para a hipertensão arterial, angina de peito e patologia músculo-esquelética, segundo alguns autores). Qualquer uma destas questões terá capacidade de aumentar o absentismo e o turnover no posto de trabalho ${ }^{4}$.

\section{Medidas de proteção coletiva e individual}

Nenhum dos artigos consultados abordou de forma explícita medidas de proteção coletivas e individuais.

Entre as primeiras poderíamos salientar a rotatividade de tarefas e a existência de sistemas de ventilação/ extração que permitissem atenuar a concentração de alguns agentes químicos, uso de produtos o menos tóxico possíveis, acesso a bancos altos que permitissem alternar entre sentado e de pé, uso de materiais de construção que atenuem o ruído e proporcionar a melhor manutenção das máquinas de forma a atenuar a produção deste e o risco de acidente. 
A nível de equipamentos de proteção individual poder-se-á realçar a existência de farda, máscara/ viseira, manguitos e luvas.

\section{CONCLUSÃO}

A bibliografia consultada deu algum destaque para as alterações hematológicas (linfomas e leucemias); neuropatias e parésias; hepatoxicidade; dermatites; insuficiência renal; asma e apneia do sono; hipoacusia; discromotopsia/ maculopatia; bem como Síndroma do Túnel Cárpico ou do Canal de Guyon, tendinites e tenossinovites, síndroma de Raynaud e doenças de Kienbock, de Quervain e Dupuytren.

Seria pertinente desenvolver investigações que avaliassem a realidade nacional.

\section{CONFLITOS DE INTERESSE, QUESTÕES ÉTICAS E/OU LEGAIS}

Nada a declarar.

\section{AGRADECIMENTOS}

Nada a declarar.

\section{BIBLIOGRAFIA}

1)Febriana $S$, Soebono $H$, Coenraads $P$. Occupational skin hazards and prevalence of occupational skin diseases in shoe manufacturing workers in Indonesia. International Archives of Occupational Health. 2014, 87, 185-194.

2)Silva K, Coelho B, Junior J, Faria L, Dutra L, Alvarenga M et al. The footwear factory's assembly sector: opposing organizational structure and quality from the ergonomic work analysis. Work 41. 2012, 1683-1690.

3)Renner I, Guimarães L, Oliveira P. A socio-technical approach for improving a brazilin shoe manufacturing system. Work 41. 2012, 1743-1750.

4)Minh K. Work-related depression and associated factors in a shoe manufacturing factory in Haiphong city, Vietnam. International Journal of Occupational Medicine and Environmental Health. 2014, 27(6), 950-958.

5)Ferreira A. Avaliação das alterações respiratórias induzidas por exposições ocupacionais através de metodologia não invasiva. Tese de Doutoramento em Ciências da Saúde, Ramo Medicina, Especialidade de Sociologia Médica, Área de Medicina Preventiva e Comunitária. Universidade de Coimbra. 2014, 1-304. 
6)Veisi H, Choobineh A, Ghaem H. Musculoskeletal problems in Iranian Hand-woven shoo-sole making operation and developing guidelines for workstation design. Theijoem. 2016, 7(2), 87-97.

7)Lima J, Colaço G, Masculo F. Members of the human body prone to musculoskeletal damages: a comparison between the sector of transportation and footwear's production. Work 41. 2012, 1582-1587.

8)Tonini S, Candura S, Lanfranco A, Mennoia V. Carpometacarpal subchondral cysts due to repetitive movements in shoemaker: a case report. International Journal of Occupational Medicine and Environmental Health. 2011, 24(4), 414-417.

Fluxograma da $1^{a}$ fase

Fluxograma da $2^{\mathrm{a}}$ fase

Tabela 1: Caraterização metodológica dos artigos selecionados

\begin{tabular}{|c|c|c|}
\hline Artigo & $\begin{array}{l}\text { Classificação } \\
\text { Metodológica }\end{array}$ & Resumo \\
\hline 1 & $\begin{array}{l}\text { observacional } \\
\text { analítico } \\
\text { transversal }\end{array}$ & $\begin{array}{l}\text { Neste artigo houve o objetivo de avaliar o risco dermatológico } \\
\text { em trabalhadores do setor da produção de calçado na } \\
\text { Indonésia. Os autores listaram e classificaram os agentes } \\
\text { químicos utilizados e posteriormente aplicaram o Questionário } \\
\text { Nórdico Ocupacional Dermatológico (2002). A amostra incluiu } \\
540 \text { indivíduos. Concluiu-se que na empresa em questão } \\
\text { existia uma exposição prolongada e repetida a agentes } \\
\text { químicos dermatologicamente nocivos. }\end{array}$ \\
\hline 2 & $\begin{array}{l}\text { estudo } \\
\text { descritivo }\end{array}$ & $\begin{array}{l}\text { Neste artigo é realizada uma análise da organização geral da } \\
\text { indústria do calçado no Brasil, destacando-se os princípios de } \\
\text { Taylor e Ford na organização do trabalho, ou seja, a } \\
\text { especialização da tarefa }\end{array}$ \\
\hline 3 & $\begin{array}{l}\text { estudo } \\
\text { experimental }\end{array}$ & $\begin{array}{l}\text { Trata-se de uma intervenção macro-ergonómica para melhorar } \\
\text { a qualidade vida e produtividade dos funcionários da indústria } \\
\text { do calçado, através da optimização do processo produtivo. }\end{array}$ \\
\hline 4 & $\begin{array}{l}\text { observacional } \\
\text { analítico } \\
\text { transversal }\end{array}$ & $\begin{array}{l}\text { Nesta investigação pretendeu-se determinar a prevalência de } \\
\text { Síndroma Depressiva e eventuais fatores etiológicos, entre } \\
\text { funcionários do setor da produção de calçado, numa cidade } \\
\text { do Vietnam. A amostra incluiu } 420 \text { funcionários e foi utilizado o } \\
\text { DSM IV. Concluiu-se que o fortalecimento da rede de suporte } \\
\text { laboral social atenua o risco de depressões. }\end{array}$ \\
\hline
\end{tabular}


Nesta tese de Doutoramento o autor avaliou alguns parâmetros respiratórios não invasivos em bombeiros, trabalhadores da indústria do calçado e da produção de plástico, de forma a tentar identificar alterações precoces fisiopatológicas antes de um quadro de patologia irreversível se instalar. $\mathrm{O}$ autor concluiu que estes parâmetros, em conjunto com a avaliação clássica (espirometria e imagiologia) podem ser muito relevantes para a medicina preventiva ocupacional.

Este trabalho teve como objetivo a quantificação da prevalência de sintomas músculo-esqueléticos nos trabalhadores que produzem calçado manualmente, no Irão, avaliando questões posturais e ergonómicas, na tentativa de desenvolver guidelines orientadoras. A amostra incluiu 240 indivíduos e foram usados a Questionário nórdico Músculoesquelético e a Metodologia Rula. Os autores concluíram que a prevalência e gravidade deste tipo de patologia é elevada neste setor profissional, justificando tal sobretudo com questões ergonómicas.

Esta investigação pretendeu avaliar detalhes associados às alterações músculo-esqueléticas que podem surgir em alguns contextos laborais, comparando o setor dos transportes e da produção de calçado entre 2008 e 2009. A amostra incluiu 227 trabalhadores e foi utilizado o diagrama de Corlett e Manenica. Os autores concluíram que os sintomas mais frequentes eram a cervicalgia, dorsalgia, omalgia e a dor nos membros inferiores; geralmente causados por posturas inapropriadas, movimentos repetitivos e postos ergonomicamente desadequados.

$8 \quad$ estudo de caso
Neste artigo os autores, para além de descreverem um caso clínico, fazem uma introdução narrativa relativa aos quistos próximos do carpo e eventual associação destes com as vibrações e os movimentos repetitivos laborais, em função das diversas teorias existentes. Concluiu-se que a imagiologia radiográfica é muito útil na abordagem inicial destas questões. Esta investigação pretendeu avaliar detalhes associados às alterações músculo-esqueléticas que podem surgir em alguns contextos laborais, comparando o setor dos transportes e da produção de calçado entre 2008 e 2009. A amostra incluiu 227 trabalhadores e foi utilizado o diagrama de Corlett e Manenica. Os autores concluíram que os sintomas mais frequentes eram a cervicalgia, dorsalgia, omalgia e a dor nos membros inferiores; geralmente causados por posturas inapropriadas, movimentos repetitivos e postos ergonomicamente desadequados.

(1)Licenciada em Medicina; Especialista em Medicina Geral e Familiar; Mestre em Ciências do Desporto; Especialista em Medicina do Trabalho; Presentemente a exercer nas empresas Medicisforma, Clinae, Servinecra, Serviço Intermédico, Securilabor, Gliese e CSW; Diretora Clínica da empresa Quercia; Diretora da 
Revista Portuguesa de Saúde Ocupacional on line. Endereços para correspondência: Rua Agostinho Fernando Oliveira Guedes, 42 4420-009 Gondomar; s_monica_santos@hotmail.com.

(2)Doutorado em Enfermagem; Mestre em Enfermagem Avançada; Especialista em Enfermagem Comunitária; Pós-graduado em Supervisão Clínica e em Sistemas de Informação em Enfermagem; Docente na Escola de Enfermagem (Porto), Instituto da Ciências da Saúde da Universidade Católica Portuguesa; Diretor Adjunto da Revista Portuguesa de Saúde Ocupacional on line. E-mail: aalmeida@porto.ucp.pt.

Santos M, Almeida A. Indústria do Calçado: principais Fatores de Risco e Riscos Laborais, Doenças Profissionais associadas e Medidas de Proteção recomendadas. Revista Portuguesa de Saúde Ocupacional on line. 2018, volume 5, s76-s85. DOI: 10.31252/RPSO.08.01.2018 